Journal of Advanced Research in Fluid Mechanics and Thermal Sciences

\title{
Numerical Investigation at Lower Stagnation Point Flow Over a Horizontal Circular Cylinder of Brinkman-Viscoelastic Fluid
}

\author{
Siti Farah Haryatie Mohd Kanafiah ${ }^{1,2}$, Abdul Rahman Mohd Kasim ${ }^{1,}{ }^{*}$, Syazwani Mohd Zokri ${ }^{3}$, Nur \\ Syamilah Arifin ${ }^{4}$ \\ Universiti Malaysia Pahang, 26300 Kuantan, Pahang, Malaysia \\ Universiti Teknologi MARA, 18500 Machang, Kelantan, Malaysia \\ Universiti Teknologi MARA, 21080 Kuala Terengganu, Terengganu, Malaysia \\ Universiti Teknologi MARA, 81570 Masai, Johor, Malaysia
}

\section{ARTICLE INFO}

\section{Article history:}

Received 10 May 2021

Received in revised form 7 August 2021

Accepted 12 August 2021

Available online 18 September 2021

\section{Keywords:}

Lower stagnation point; horizontal circular cylinder; fluid flow; porous medium

\section{ABSTRACT}

Investigations on the characteristics of fluid flow in manufacturing processes are essential since it will determine the quality of the end products. The flow might be involved whether the Newtonian (viscous) or non-Newtonian fluid moving over the different body depending on the process activities. Since the experimental works sometimes costly and hazardous, the study via mathematical approach is necessary to counter the limitations. Hence, this paper aims to investigate the flow at lower stagnation point over a horizontal circular cylinder on Brinkman Viscoelastic fluid embedded in porous medium. Mathematical model is constructed in terms of partial differential equations with some physical conditions to represent the condition of the problem. An appropriate non-dimensional variable is introduced to transform the model into the solvable system, which is in less complexity, and then the system is solved using the Runge-Kutta-Fehlberg method. The numerical solutions for the temperature and velocity profiles as well as skin friction and heat transfer coefficient are computed and presented in graphical and tabular form. The feature of the flow and heat transfer characteristics for various values of mixed convection, Brinkman and viscoelastic parameter are analysed and discussed. This study has found that the incremented Brinkman and viscoelastic parameter have declined the fluid velocity while opposite trend is observed for temperature distribution. The theoretical results produced are relevance to researchers and engineers. It can be used for comparative purposes in data validation or experimentation study.

\section{Introduction}

The convective of heat transfer in porous medium has been a topic of interest by numerous industrial applications. For example, the applications in various discipline such as the packed sphere beds modelling, the coating of wires and petroleum reservoirs. The understanding of heat transfer and characteristic of fluid flow are very important to produce the desirable output. About a dozen

\footnotetext{
* Corresponding author.

E-mail address: rahmanmohd@ump.edu.my
}

https://doi.org/10.37934/arfmts.87.2.5665 
articles have been published on convective boundary layer problem from different surface geometry, for instance Arifin et al., [1], Dasman et al., [2], Kasim et al., [3], Zokri et al., [4], Nazar et al., [5], Anwar et al., [6], Tham et al., [7], Kasim and Shafie [8], and Tlili [9].

In general, the lower stagnation point flow over a horizontal circular cylinder occurs when the vertical flow first reaches the bottom of the cylinder. The demand for mixed convection in industrial processes has increased tremendously over the last decade. The effect of the buoyancy force becomes significant due to the temperature difference that is described by mixed convection flow. There have been many studies focusing on the flow of mixed convection over a horizontal circular cylinder. Nazar et al., [10] investigated how the control parameters give impact to the characteristics of fluid flow and heat transport. Subsequently, Tham et al., [11] used the Buongiorno-Darcy model to investigate the flow of both heated and cooled cylinder embedded in a porous medium filled with nanofluid. The research on the flow over a horizontal circular cylinder was then improvised by Zokri et al., [12] where the model of Jeffrey nanofluid is deliberated. Anwar et al., [13] examined the flow in viscoelastic fluid at constant temperature, whereas Ishak et al., [14] found the dual solutions in flow of micropolar fluid. Moreover, Aziz et al., [15] studied the flow of viscoelastic micropolar fluid flow with the effect of magneto-hydrodynamic. Later on, Aziz et al., [16] concentrated on the development model of viscoelastic fluid with the influence of microrotation. Besides, Mahat et al., [17] investigated the flow in viscoelastic nanofluid by considering constant wall temperature. Under the similar problem, Mahat et al., [18] concentrated on the heat generation and flow of thermal performance with convective boundary condition.

Nowadays, the flows through porous medium have gain attention in industrial and engineering applications such as filtration process, drying of porous materials and purification process. The Brinkman model is one of the classical models that have been considered in porous medium for high porosity flow [19-22].

Inspired by the study in literature, this study aims to investigate the flow at lower stagnation point by considering the mixed convection Brinkman Viscoelastic model. The solutions are attained using the Runge-Kutta-Fehlberg-Method and the analysis focused on the effects of mixed convection, brinkman and viscoelastic parameters over velocity and temperature profiles.

\section{Mathematical Formulation}

Present investigation considers the steady mixed convection boundary layer flow past a horizontal circular cylinder of radius $a$ embedded in a porous medium. It is stated that $T_{w}$ and $T_{\infty}$ are the constant surface and ambient temperature, respectively, where $T_{w}>T_{\infty}$ represents the assisting flow and $T_{w}<T_{\infty}$ indicates opposing flow. The free stream velocity, $\frac{1}{2} U_{\infty}$ is moving vertically upwards over the cylinder and the gravitational acceleration is denoted as $g$. Figure 1 shows the physical model of the problem and coordinate system. From the lower stagnation point, $\bar{x}$ and $\bar{y}$ are measured which is $\bar{x}=0$ and $\bar{y}$ is normal to the cylinder surface. 


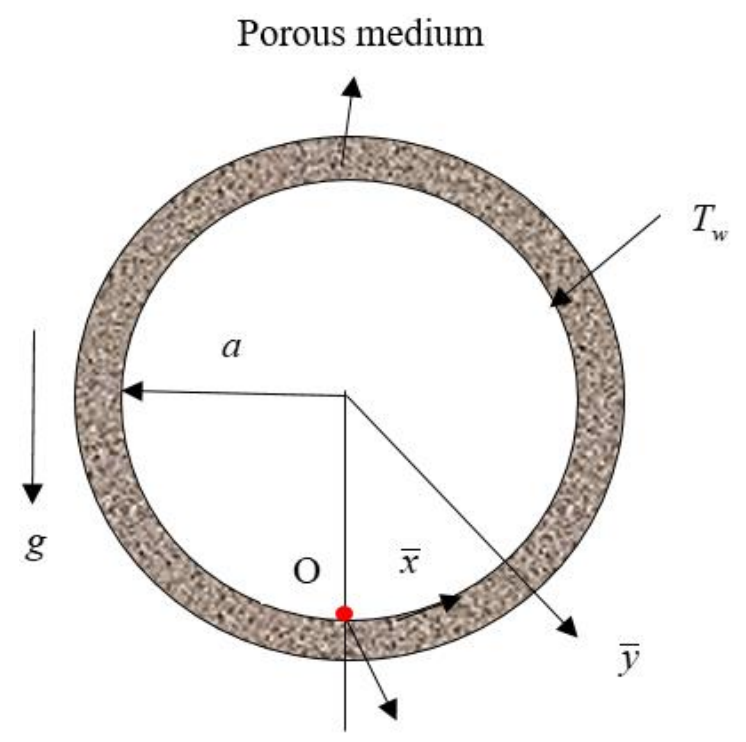

Lower Stagnation Point

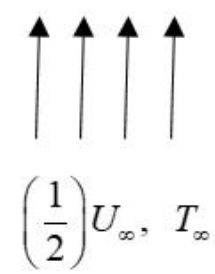

Fig. 1. Physical model and coordinate system

The governing equations under the boundary layer approximation are

$\frac{\partial \bar{u}}{\partial \bar{x}}+\frac{\partial \bar{v}}{\partial \bar{y}}=0$

$$
\begin{aligned}
\frac{\mu}{K} \bar{u}= & -\frac{\partial p}{\partial \bar{x}}+\frac{\mu}{\phi}\left(\frac{\partial^{2} \bar{u}}{\partial \bar{x}^{2}}+\frac{\partial^{2} \bar{u}}{\partial \bar{y}^{2}}\right) \\
& +k_{0}\left[\begin{array}{l}
\left.\bar{u}\left(\frac{\partial^{3} \bar{u}}{\partial \bar{x}^{3}}+\frac{\partial^{3} \bar{u}}{\partial \bar{x} \partial \bar{y}^{2}}\right)+\bar{v}\left(\frac{\partial^{3} \bar{u}}{\partial \bar{x}^{2} \partial \bar{y}}+\frac{\partial^{3} \bar{u}}{\partial \bar{y}^{3}}\right)-\frac{\partial \bar{u}}{\partial \bar{y}}\left(\frac{\partial^{2} \bar{u}}{\partial \bar{x} \partial \bar{y}}+\frac{\partial^{2} \bar{v}}{\partial \bar{x}^{2}}\right)\right]-\rho g \sin (\bar{x} / a), \\
+\frac{\partial \bar{u}}{\partial \bar{x}}\left(3 \frac{\partial^{2} \bar{v}}{\partial \bar{x} \partial \bar{y}}+\frac{\partial^{2} \bar{u}}{\partial \bar{y}^{2}}\right)-2 \frac{\partial \bar{v}}{\partial \bar{x}} \frac{\partial^{2} \bar{u}}{\partial \bar{x} \partial \bar{y}}
\end{array}\right]
\end{aligned}
$$

$$
\begin{aligned}
\frac{\mu}{K} \bar{v}=- & -\frac{\partial p}{\partial \bar{y}}+\frac{\mu}{\phi}\left(\frac{\partial^{2} \bar{v}}{\partial \bar{x}^{2}}+\frac{\partial^{2} \bar{v}}{\partial \bar{y}^{2}}\right) \\
& +k_{0}\left[\begin{array}{l}
\left.\bar{u}\left(\frac{\partial^{3} \bar{v}}{\partial \bar{x}^{3}}+\frac{\partial^{3} \bar{v}}{\partial \bar{x} \partial \bar{y}^{2}}\right)+\bar{v}\left(\frac{\partial^{3} \bar{v}}{\partial \bar{x}^{2} \partial \bar{y}}+\frac{\partial^{3} \bar{v}}{\partial \bar{y}^{3}}\right)-\frac{\partial \bar{v}}{\partial \bar{x}}\left(\frac{\partial^{2} \bar{v}}{\partial \bar{x} \partial \bar{y}}+\frac{\partial^{2} \bar{u}}{\partial \bar{y}^{2}}\right)\right]+\rho g \cos (\bar{x} / a), \\
-\frac{\partial \bar{v}}{\partial \bar{y}}\left(3 \frac{\partial^{2} \bar{v}}{\partial \bar{x} \partial \bar{y}}-\frac{\partial^{2} \bar{v}}{\partial \bar{x}^{2}}\right)-2 \frac{\partial \bar{u}}{\partial \bar{y}} \frac{\partial^{2} \bar{v}}{\partial \bar{x} \partial \bar{y}}
\end{array}\right]
\end{aligned}
$$

$\bar{u} \frac{\partial T}{\partial \bar{x}}+\bar{v} \frac{\partial T}{\partial \bar{y}}=\alpha_{m}\left(\frac{\partial^{2} T}{\partial \bar{x}^{2}}+\frac{\partial^{2} T}{\partial \bar{y}^{2}}\right)$ 
with the boundary conditions

$\bar{v}=0, \quad \bar{u}=0, \quad T=T_{w}, \quad$ at $\quad \bar{y}=0$,

$\bar{u} \rightarrow \bar{u}_{e}(\bar{x}), \quad \bar{v} \rightarrow 0, \quad T \rightarrow T_{\infty} \quad$ as $\quad \bar{y} \rightarrow \infty$.

for which $\rho=\rho_{\infty}\left[1-\beta\left(T-T_{\infty}\right)\right]$ and the components of velocity along $\bar{x}$ and $\bar{y}$ axes are stated as $\bar{u}$ and $\bar{v}$ respectively. Next, $\mu, K, \phi, k_{0}, \rho, p, \beta, T$, and $\alpha_{m}$ are the respective dynamic viscosity, permeability of porous medium, viscoelasticity, fluid density, pressure, thermal expansion coefficient, fluid temperature, and effective thermal diffusivity of porous. The external velocity flow is denoted as $\bar{u}_{e}(\bar{x})=U_{\infty} \sin (\bar{x} / a)$. Eq. (1) to (5) are transformed into dimensionless form by introducing the non-dimensional variables as follows

$x=\bar{x} / a, \quad y=P e^{1 / 2}(\bar{y} / a), \quad u=\bar{u} / U_{\infty}, \quad v=P e^{1 / 2}\left(\bar{v} / U_{\infty}\right)$,

$\theta=\left(T-T_{\infty}\right) /\left(T_{w}-T_{\infty}\right), \quad u_{e}(\bar{x})=\bar{u}_{e}(\bar{x}) / U_{\infty}$,

where $P e=U_{\infty} a / \alpha_{m}$ is modified Péclet number for porous medium. From Eq. (2) and (3), pressure, $p$ is eliminated and the boundary layer approximation $P e \rightarrow \infty$ is considered. Substituting Eq. (6) into Eq. (1) to (5) yield

$\frac{\partial u}{\partial x}+\frac{\partial v}{\partial y}=0$

$\frac{\partial u}{\partial y}=\Gamma \frac{\partial^{3} u}{\partial y^{3}}+k_{1}\left[\begin{array}{l}u \frac{\partial^{4} u}{\partial x \partial y^{3}}+\frac{\partial^{3} u}{\partial x \partial y^{2}} \frac{\partial u}{\partial y}+v \frac{\partial^{4} u}{\partial y^{4}}+\frac{\partial^{3} u}{\partial y^{3}} \frac{\partial v}{\partial y}-\frac{\partial u}{\partial y} \frac{\partial^{3} u}{\partial x \partial y^{2}} \\ -\frac{\partial^{2} u}{\partial x \partial y} \frac{\partial^{2} u}{\partial y^{2}}+\frac{\partial u}{\partial x} \frac{\partial^{3} u}{\partial y^{3}}+\frac{\partial^{2} u}{\partial y^{2}} \frac{\partial^{2} u}{\partial x \partial y}\end{array}\right]+\lambda \frac{\partial \theta}{\partial y} \sin x$,

$u \frac{\partial \theta}{\partial x}+v \frac{\partial \theta}{\partial y}=\frac{\partial^{2} \theta}{\partial y^{2}}$

$u=0, \quad v=0, \quad \theta=1 \quad$ at $\bar{y}=0$,

$u \rightarrow u_{e}, v \rightarrow 0, \quad \theta \rightarrow 0 \quad$ as $\bar{y} \rightarrow \infty$,

Integrating Eq. (8) and applying the similarity transformation variables

$\psi=x f(x, y), \quad \theta=\theta(x, y)$,

where $\psi$ implies the stream function while $\theta$ is the temperature of fluid defined as $u=\frac{\partial \psi}{\partial y}$ and $v=-\frac{\partial \psi}{\partial x}$. Eq. (7) is completely fulfilled. Substituting Eq. (11) into Eq. (8), (9), and (10) lead to 


$$
\begin{aligned}
& f^{\prime}= \Gamma f^{\prime \prime \prime}+k_{1}\left[x f^{\prime} \frac{\partial f^{\prime \prime \prime}}{\partial x}+2 f^{\prime \prime \prime \prime}-x \frac{\partial f}{\partial x} f^{(i v)}-f f^{(i v)}-x f^{\prime \prime} \frac{\partial f^{\prime \prime}}{\partial x}-\left(f^{\prime \prime}\right)^{2}+x \frac{\partial f^{\prime}}{\partial x} f^{\prime \prime \prime}\right] \\
&+(1+\lambda \theta) \frac{\sin x}{x}, \\
& \theta^{\prime \prime}+f \theta^{\prime}=x\left(f^{\prime} \frac{\partial \theta}{\partial x}-\frac{\partial f}{\partial x} \theta^{\prime}\right) . \\
& f(x, 0)=0, \quad f^{\prime}(x, 0)=0, \quad \theta(x, 0)=1, \quad \text { at } y=0, \\
& f^{\prime}(x, \infty) \rightarrow \frac{\sin x}{x}, \quad f^{\prime \prime}(x, \infty) \rightarrow 0, \quad \theta(x, \infty) \rightarrow 0 \quad \text { as } y \rightarrow \infty .
\end{aligned}
$$

At the lower stagnation point of circular cylinder $(x \approx 0)$ Eq. (12) to (14) are reduced to ordinary differential equations as follows

$$
\begin{aligned}
& f^{\prime}-\Gamma f^{\prime \prime \prime}-k_{1}\left[2 f^{\prime} f^{\prime \prime \prime}-f f^{(i v)}-\left(f^{\prime \prime}\right)^{2}\right]-1-\lambda \theta=0, \\
& \theta^{\prime \prime}+f \theta^{\prime}=0 .
\end{aligned}
$$

subjected to boundary condition

$$
\begin{aligned}
& f(0)=0, \quad f^{\prime}(0)=0, \quad \theta(0)=1 \\
& f^{\prime}(\infty) \rightarrow 1, \quad f^{\prime \prime}(\infty) \rightarrow 0, \quad \theta(\infty) \rightarrow 0
\end{aligned}
$$

where primes represent the differentiation with respect to $y, \Gamma=\frac{D a}{\phi} P e$ is Brinkman parameter, $D a=\frac{K}{a^{2}}$ is Darcy number, $\lambda=\frac{R a}{P e}$ is mixed convection parameter, $R a=\frac{g K \beta\left(T_{w}-T_{\infty}\right) a}{\alpha_{m} v}$ is Rayleigh number, and $\mathrm{k}_{1}=\frac{k_{0} K U_{\infty} P e}{\mu a^{3}}$ is Viscoelastic parameter.

\section{Results and Discussion}

The Eq. (15) and (16) with boundary conditions (Eq. (17)) were solved numerically using RungeKutta-Fehlberg-Method for various values of mixed convection parameter, $\lambda$, Brinkman parameter, $\Gamma$, and Viscoelastic parameter, $k_{1}$, which is programmed in Maple software. In this study, the boundary layer thickness in the range of 4 to 10 are used to fulfill boundary conditions asymptotically. The fixed parameters of mixed convection used in the simulations are $\lambda=1$ for assisting flow, except otherwise stated. For validation purposes, the comparison of the present study has been made with the existing publication documented by Nazar et al., [23] as depicted in Table 1. The result shows a strong agreement, and this gives the authors confidence that the computation proposed is accurate.

In Figure 2, the behaviour of velocity $f^{\prime}(\eta)$ and temperature profile $\theta(\eta)$ of fluid were observed with changes in the value of $\Gamma$. It is noticed that in Figure 2(a), the velocity profile decreases as $\Gamma$ increases due to drag force improvement. Further, increasing the value of $\Gamma$ in Figure 2 (b) growth 
the temperature profile accordingly. Note that when the thickness of thermal boundary layer is increased due to $\Gamma$, the temperature at the wall is high.

\section{Table 1}

Comparison between the present result and the existing publications for values of $f^{\prime \prime}(0)$ and $-\theta^{\prime}(0)$ with $\Gamma=0.1, k_{1} \rightarrow 0$ (very small) and various value of $\lambda=1$

\begin{tabular}{lllll}
\hline$\lambda$ & Nazar et al., [23] & $-\theta^{\prime}(0)$ & Present & $-\theta^{\prime}(0)$ \\
\hline 0.5 & $f^{\prime \prime}(0)$ & 0.7240 & $f^{\prime \prime}(0)$ & 0.7239 \\
1 & 4.3999 & 0.3999 & 0.7790 \\
2 & 5.5923 & 0.8791 & 5.5922 & 0.8705 \\
3 & 7.8768 & 0.9460 & 7.8767 & 0.9458 \\
\hline
\end{tabular}

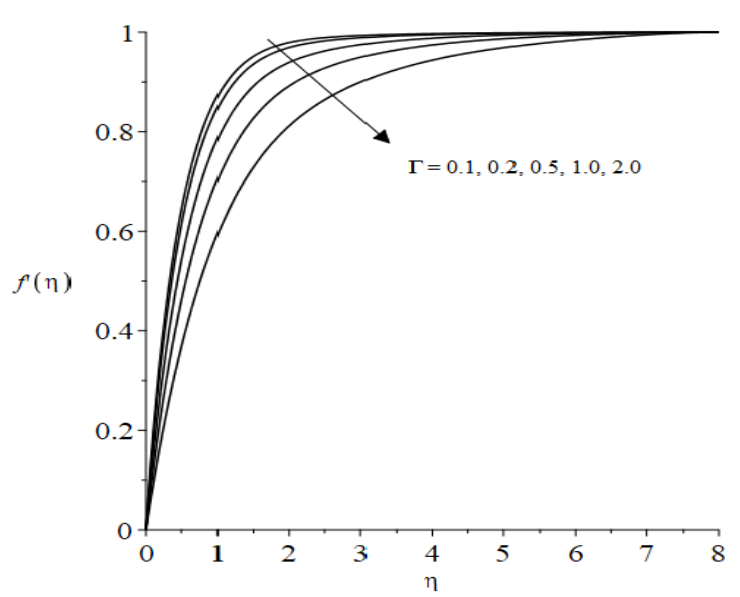

(a)

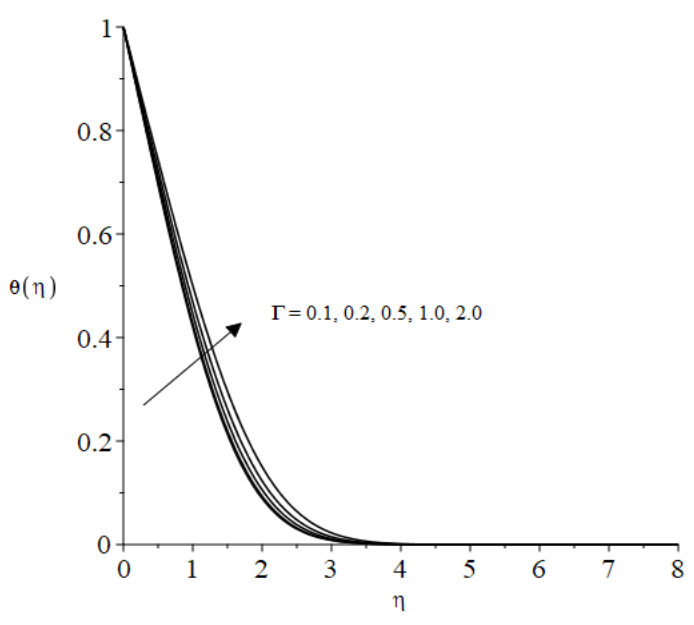

(b)

Fig. 2. Effect of $\Gamma$ on $f^{\prime}(\eta)$ and $\theta(\eta)$

Figure 3 illustrates how the viscoelastic parameter $k_{1}$ influences the fluid of temperature and velocity profiles. Based on Figure 3(a), for larger $k_{1}$, the velocity of the fluid is seen to decrease. It indicates that the velocity slows down as the viscosity increases. Meanwhile, in Figure 3(b), the temperature profile shows the reverse trend for the increment of $k_{1}$. This phenomenon physically happens due the viscosity and elasticity properties.

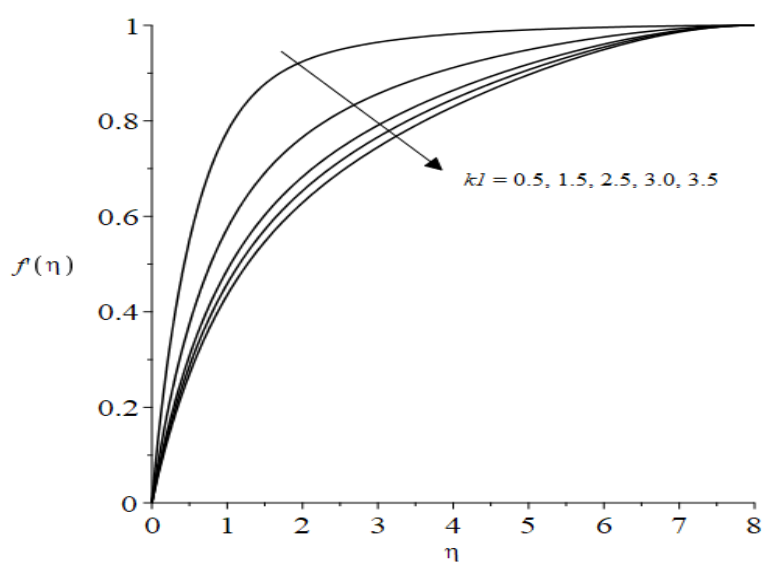

(a)

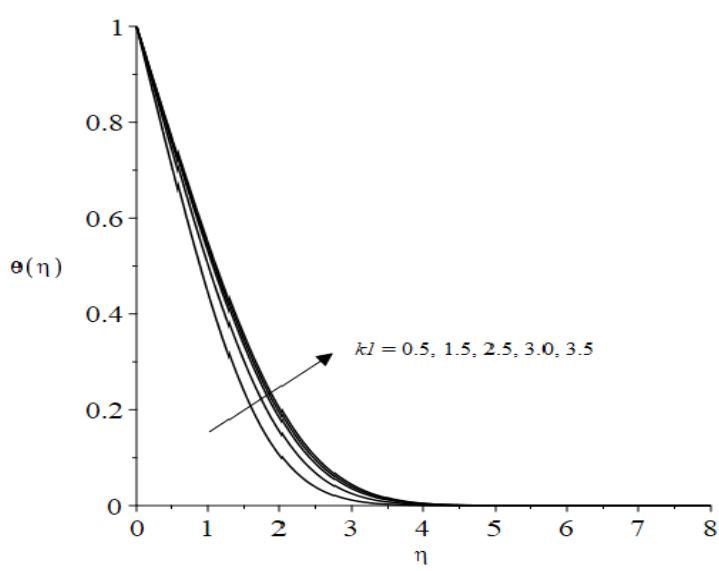

(b)

Fig. 3. Effect of $k_{1}$ on $f^{\prime}(\eta)$ and $\theta(\eta)$ 
Figure $4(a)$ depicts the nature of varying $\lambda$ values in velocity and temperature profiles. The velocity profile is observed to boosts as $\lambda$ grows. Owing to favorable buoyancy effects, the fluid velocity rises within the boundary layer flow. It is also identified that the velocity profile is overrun for high $\lambda$. Contrary trends have been observed for temperature profile. Figure 4 (b) shows the reduction of convective heat transfer as the $\lambda$ increases, thus lowering the temperature.

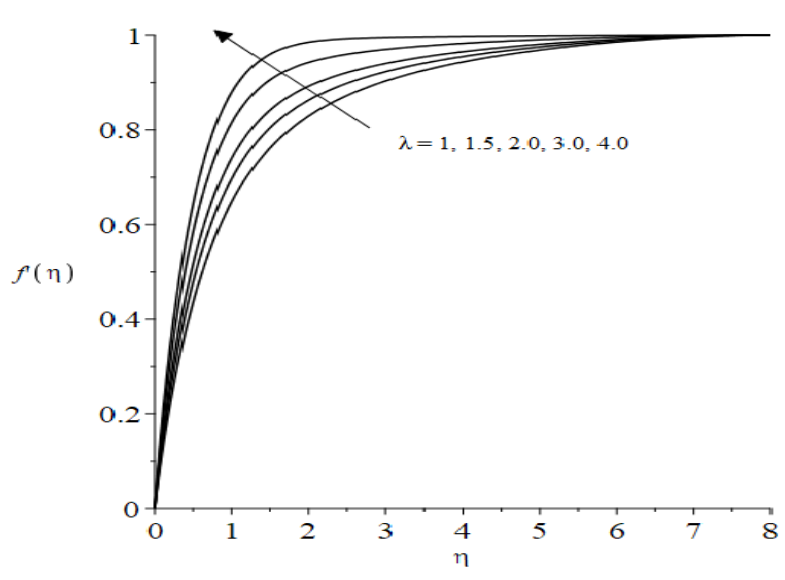

(a)

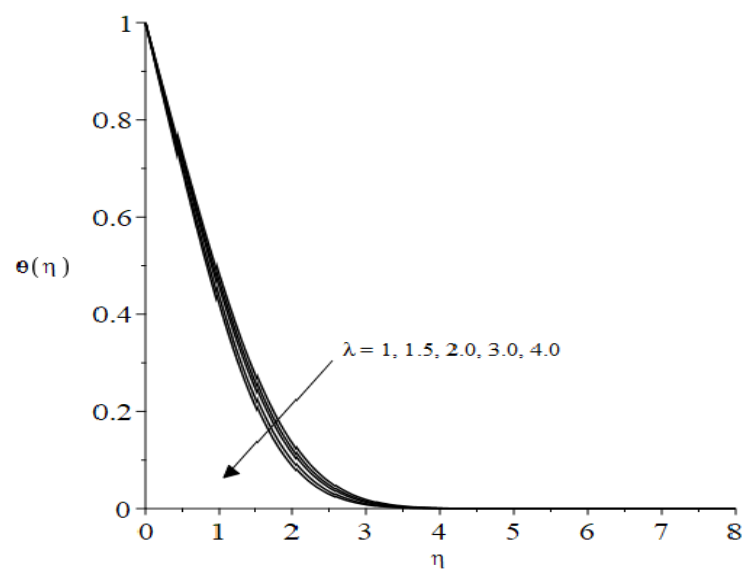

(b)

Fig. 4. Effect of $\lambda$ on $f^{\prime}(\eta)$ and $\theta(\eta)$

Figure 5 displays the behavior of velocity profile for both Brinkman and Brinkman viscoelastic fluid when $\lambda=1$. It is observed that for Brinkman fluid, there exist an acceleration of the velocity profile compared to Brinkman viscoelastic fluid. It can be concluded that the velocity profile for Brinkman viscoelastic fluid is slower than the Brinkman fluid. This phenomena clearly shows the significance of fluid viscosity that will affect the velocity profile.

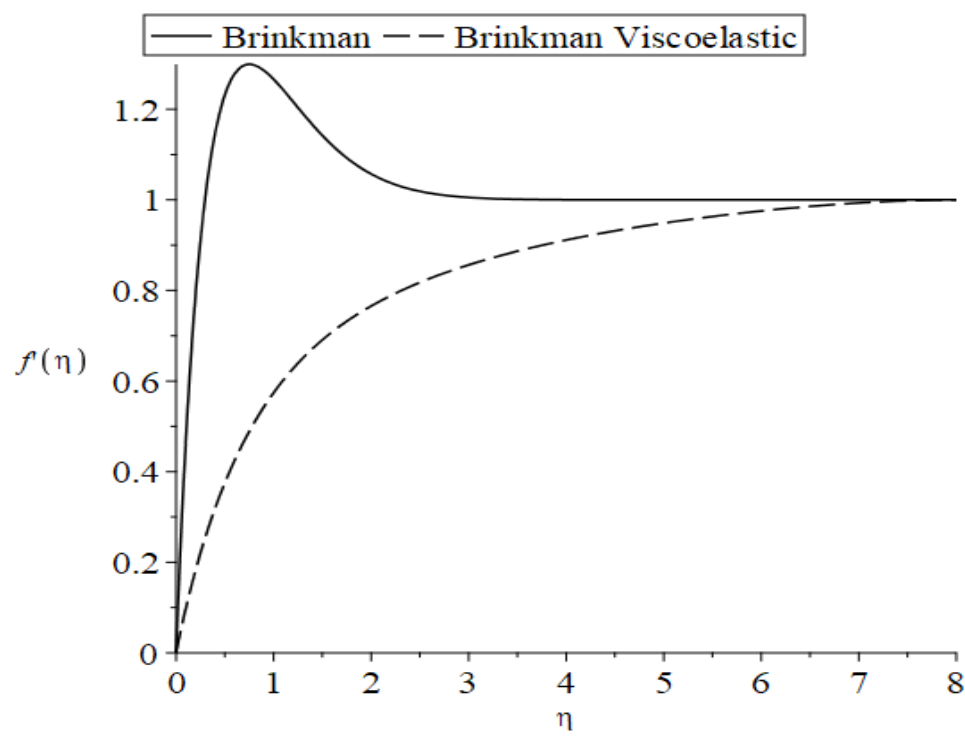

Fig. 5. Comparison between Brinkman fluid and Brinkman viscoelastic fluid on $f^{\prime}(\eta)$ for $\lambda=1$

Table 2 tabulates several parameters on the numerical values of skin friction and heat transfer coefficient. It is described that, for larger values of $\lambda$, the skin friction, and heat transfer will boost up. The increase in buoyancy force accelerates the fluid velocity as well as skin friction of the fluid. In 
contrast, the decrease in thermal boundary layer thickness as can be seen in Figure 4(b) will increase the heat transfer. Meanwhile, as $\Gamma$ and $k_{1}$ increase, the skin friction is decreased and weak heat transfer is detected.

\section{Table 2}

\begin{tabular}{ccccc}
\multicolumn{4}{c}{ Numerical values of $f^{\prime \prime}(0)$ and $-\theta(0)$ for various $\lambda, \Gamma$ and $k_{1}$} \\
\hline$\lambda$ & $\Gamma$ & $k_{1}$ & $f^{\prime \prime}(0)$ & $-\theta(0)$ \\
\hline 1.0 & 0.1 & 1.0 & 1.30573 & 0.53880 \\
1.5 & & & 1.46679 & 0.55550 \\
2.0 & & & 1.61300 & 0.57003 \\
3.0 & & & 1.87362 & 0.59458 \\
4.0 & & & 2.10400 & 0.61499 \\
1.0 & 0.1 & 0.3 & 2.17106 & 0.61492 \\
& 0.2 & & 1.95391 & 0.60507 \\
& 0.5 & & 1.57133 & 0.58138 \\
& 1.0 & & 1.24294 & 0.55292 \\
& 2.0 & & 0.92806 & 0.51575 \\
1.0 & 0.1 & 0.5 & 1.76434 & 0.58300 \\
& & 1.5 & 1.08638 & 0.51297 \\
& & 2.5 & 0.85652 & 0.48091 \\
& & 3.0 & 0.78582 & 0.46966 \\
& & 3.5 & 0.73029 & 0.46025 \\
\hline
\end{tabular}

\section{Conclusions}

The problem of steady mixed convection boundary layer flow at lower stagnation points of a horizontal circular cylinder embedded in porous medium under the Brinkman Viscoelastic fluid model has been investigated numerically. It can be inferred that the principle of Brinkman theory and viscoelastic knowledge give a significance effect to the fluid flow characteristics. In general, increasing the Brinkman and viscoelastic parameters will retard the fluid velocity. Oppositely, the temperature distribution increases owing to increment in Brinkman and viscoelastic parameter.

\section{Acknowledgement}

The authors would like to acknowledge The Ministry of Higher Education and Universiti Malaysia Pahang for the financial support through PGRS2003169 and The Ministry of Higher Education and Universiti Malaysia Pahang for the financial support through RDU190303 \& The Fundamental Research Grant Scheme for Research Acculturation of Early Career Researchers (FRGS-RACER) (Ref: RACER/1/2019/STG06/UMP//1) through RDU192602. Deep appreciation also extended to Universiti Teknologi MARA Cawangan Kelantan for the guidance and support.

\section{References}

[1] Arifin, Nur Syamilah, Syazwani Mohd Zokri, Abdul Rahman Mohd Kasim, Mohd Zuki Salleh, and Nurul Farhain Mohammad. "Numerical solutions of the aligned magnetic field on the boundary layer flow and heat transfer over a stretching sheet by using Keller-box method." In The National Conference for Postgraduate Research, pp. 266274. 2016.

[2] Dasman, Anisah, Abdul Rahman Mohd Kasim, Nurul Farahain Mohammad, Aurangzaib Mangi, and Sharidan Shafie. "Mixed convection boundary layer flow of viscoelastic fluids past a sphere." In Defect and Diffusion Forum, vol. 336, pp. 57-63. Trans Tech Publications Ltd, 2013. https://doi.org/10.4028/www.scientific.net/DDF.336.57

[3] Kasim, Abdul Rahman Mohd, Lim Yeou Jiann, Sharidan Shafie, and Anati Ali. "The effects of heat generation or absorption on MHD stagnation point of Jeffrey fluid." In AIP Conference Proceedings, vol. 1605, no. 1, pp. $404-409$. American Institute of Physics, 2014. https://doi.org/10.1063/1.4887623 
[4] Zokri, Syazwani Mohd, Nur Syamilah Arifin, Muhammad Khairul Anuar Mohamed, Abdul Rahman Mohd Kasim, Nurul Farahain Mohammad, and Mohd Zuki Salleh. "Mathematical model of mixed convection boundary layer flow over a horizontal circular cylinder filled in a Jeffrey fluid with viscous dissipation effect." Sains Malaysiana 47, no. 7 (2018): 1607-1615. https://doi.org/10.17576/jsm-2018-4707-32

[5] Nazar, Roslinda, Norsarahaida Amin, and Ioan Pop. "Mixed convection boundary layer flow about an isothermal sphere in a micropolar fluid." International journal of thermal sciences 42, no. 3 (2003): $283-293$. https://doi.org/10.1016/S1290-0729(02)00027-3

[6] Anwar, Muhammad Imran, Sharidan Shafie, Abdul Rahman M. Kasim, and Mohd Zuki Salleh. "Radiation effect on mhd stagnation-point flow of a nanofluid over a nonlinear stretching sheet with convective boundary condition." Heat Transfer Research 47, no. 9 (2016). https://doi.org/10.1615/HeatTransRes.2016007840

[7] Tham, Leony, Roslinda Nazar, and loan Pop. "Mixed convection flow over a horizontal circular cylinder with constant heat flux embedded in a porous medium filled by a nanofluid: Buongiorno-Darcy model." Heat and Mass Transfer 52, no. 9 (2016): 1983-1991. https://doi.org/10.1007/s00231-015-1720-2

[8] Kasim, A., and S. Shafie. "Mixed Convection Boundary Layer of a Viscoelastic Fluid past a Circular Cylinder with Constant Heat Flux." In Proceedings of the 1st Regional Conference on Applied and Engineering Mathematics, vol. 1, no. 20, pp. 124-129. 2010.

[9] Tlili, Iskander. "Effects MHD and heat generation on mixed convection flow of Jeffrey fluid in microgravity environment over an inclined stretching sheet." Symmetry 11, no. $3 \quad$ (2019): 438. https://doi.org/10.3390/sym11030438

[10] Nazar, Roslinda, Norsarahaida Amin, and loan Pop. "Mixed convection boundary-layer flow from a horizontal circular cylinder in micropolar fluids: case of constant wall temperature." International Journal of Numerical Methods for Heat \& Fluid Flow (2003). https://doi.org/10.1108/09615530310456778

[11] Tham, Leony, Roslinda Nazar, and loan Pop. "Mixed convection flow from a horizontal circular cylinder embedded in a porous medium filled by a nanofluid: Buongiorno-Darcy model." International Journal of Thermal Sciences 84 (2014): 21-33. https://doi.org/10.1016/i.ijthermalsci.2014.04.020

[12] Mohd Zokri, Syazwani, Nur Syamilah Arifin, Abdul Rahman Mohd Kasim, and Mohd Zuki Salleh. "Free Convection Boundary Layer Flow of Jeffrey Nanofluid on a Horizontal Circular Cylinder with Viscous Dissipation Effect." Journal of Advanced Research in Micro and Nano Engineering 1, no. 1 (2020): 1-14.

[13] Anwar, llyana, Norsarahaida Amin, and loan Pop. "Mixed convection boundary layer flow of a viscoelastic fluid over a horizontal circular cylinder." International Journal of Non-Linear Mechanics 43, no. 9 (2008): 814-821. https://doi.org/10.1016/j.ijnonlinmec.2008.04.008

[14] Ishak, Anuar, Roslinda Nazar, and loan Pop. "Dual solutions in mixed convection boundary layer flow of micropolar fluids." Communications in Nonlinear Science and Numerical Simulation 14, no. 4 (2009): 1324-1333. https://doi.org/10.1016/i.cnsns.2008.01.017

[15] Aziz, Laila Amera, Abdul Rahman Mohd Kasim, Mohd Zuki Salleh, Sharidan Shafie, and Wan Nur Syahidah Wan Yusoff. "Boundary layer flow of mixed convection viscoelastic micropolar fluid over a horizontal circular cylinder with aligned magnetohydrodynamic effect." Malaysian Journal of Fundamental and Applied Sciences 13, no. 4 (2017): 567-571. https://doi.org/10.11113/mjfas.v0n0.590

[16] Aziz, L. A., Kasim, A. R. M. and Salleh, M. Z. "Development on mathematical model of convective boundary layer flow of viscoelastic fluid with microrotation effect under constant wall temperature thermal condition over a bluff body." ASM Science Journal 12, no. 5 (2019): 86-90.

[17] Mahat, Rahimah, Noraihan Afiqah Rawi, Abdul Rahman Mohd Kasim, and Sharidan Shafie. "Mixed convection boundary layer flow past a horizontal circular cylinder in viscoelastic nanofluid with constant wall temperature." Malaysian Journal of Fundamental and Applied Sciences 13, no. 4-1 (2017): 310-314. https://doi.org/10.11113/mjfas.v13n4-1.869

[18] Mahat, R., Rawi, N. A., Kasim, A. R. M. and Shafie, S. "Heat generation effect on mixed convection flow of viscoelastic nanofluid: convective boundary condition solution." Malaysian Journal of Fundamental and Applied Sciences 16, no. 2 (2020): 166-172. https://doi.org/10.11113/mjfas.v16n2.1367

[19] Dubey, R. and Murthy, P. V. S. N. "The onset of double-diffusive convection in a Brinkman porous layer with convective thermal boundary conditions." AIP Advances 9, no. 045322 (2019): 1-12. https://doi.org/10.1063/1.5087037

[20] Kausar, M. S., Hussanan, A., Mamat, M. and Ahmad, B. "Boundary layer flow through Darcy-Brinkman porous medium in the presence of slip effects and porous dissipation." Symmetry 11, no. 659 (2019): 1-11. https://doi.org/10.3390/sym11050659

[21] Khan, A., Khan, D., Khan, I., Ali, F., UI Karim, F. and Nisar, K. S. "MHD flow of brinkman type $\mathrm{H}_{2} \mathrm{O}-\mathrm{Cu}, \mathrm{Ag}, \mathrm{TiO}_{2}$ and $\mathrm{Al}_{2} \mathrm{O}_{3}$ nanofluids with chemical reaction and heat generation effects in a porous medium." Journal of Magnetics 24, no. 2 (2019): 262-270. https://doi.org/10.4283/JMAG.2019.24.2.262 
[22] Yadav, Dhananjay. "The onset of Darcy-Brinkman convection in a porous medium layer with vertical throughflow and variable gravity field effects." Heat Transfer 49, no. 5 (2020): 3161-3173. https://doi.org/10.1002/htj.21767

[23] Nazar, Roslinda, Norsarahaida Amin, Diana Filip, and loan Pop. "The Brinkman model for the mixed convection boundary layer flow past a horizontal circular cylinder in a porous medium." International Journal of Heat and Mass Transfer 46, no. 17 (2003): 3167-3178. https://doi.org/10.1016/S0017-9310(03)00122-4 